\title{
Biomechanical and biological performances of Diels-Alder crosslinked thermogelling
}

\section{bioink}

Lukas Hahn ${ }^{1 *}$, Matthias Beudert ${ }^{2}$, Marcus Gutmann ${ }^{2}$, Larissa Kessler ${ }^{1}$, Philipp Stahlhut ${ }^{3}$, Lena Fischer ${ }^{4}$, Thomas Lorson ${ }^{2}$, Ingo Thievessen ${ }^{4}$, Tessa Lühmann ${ }^{2}$ and Robert Luxenhofer ${ }^{1,5}$

${ }^{1}$ Institute of Pharmacy and Food Chemistry, Julius-Maximilians-University Würzburg, Am Hubland, 97074 Würzburg, Germany

${ }^{2}$ Functional Polymer Materials, Chair for Advanced Materials Synthesis, Institute for Functional Materials and Biofabrication, Department of Chemistry and Pharmacy and Bavarian Polymer Institute, Julius-MaximiliansUniversity Würzburg, Röntgenring 11, 97070 Würzburg, Germany

${ }^{3}$ Department for Functional Materials in Medicine and Dentistry, University of Würzburg, Pleicherwall 2, 97070 Würzburg, Germany

${ }^{4}$ Center for Medical Physics and Technology, Biophysics Group, Friedrich-Alexander-University of ErlangenNuremberg, Henkestrasse 91, 91052 Erlangen, Germany

${ }^{5}$ Soft Matter Chemistry, Department of Chemistry and Helsinki Institute of Sustainability Science, Faculty of Science, University of Helsinki, P.O. Box 55, FIN-00014, Helsinki, Finland

*Contributed equally

Corresponding authors: tessa.luehmann@uni-wuerzburg.de, robert.luxenhofer@helsinki.fi

\section{Keywords: bioprinting, biofabrication, hydrogels, chemical crosslinking}




\begin{abstract}
Hydrogels are key components in bioink formulations to ensure printability and stability in biofabrication. In this study a well-known post-polymerization modification approach is introduced into thermogelling diblock copolymers, comprising poly(2-methyl-2-oxazoline) and thermoresponsive poly(2-n-propyl-2-oxazine). While the thermogelling and shear-thinning properties allow excellent printability, trigger-less cell-friendly Diels-Alder click-chemistry yields long-term shape-fidelity. The introduced platform enables easy incorporation of cellbinding moieties (RGD-peptide) for cellular interaction. The hydrogel was functionalized with RGD-peptides using thiol-maleimide chemistry and growth as well as morphology of fibroblast seeded on top of the hydrogels confirmed the cell adhesion facilitated by the peptides. Finally, bioink formulations were tested for biocompatibility by incorporating fibroblasts homogenously inside polymer solution pre-printing and exhibited good cytocompatibility after the printing process and crosslinking. The established bioink system combining a two-step approach by physical precursor gelation followed by additional chemical stabilization offers a broad versatility for further biomechanical adaptation or bioresponsive peptide modification.
\end{abstract}

\title{
Introduction
}

Advances in biofabrication strongly relies on the availability of suitable bioinks. ${ }^{1-3}$ These materials, in turn, are mostly based on polymers - either of synthetic or natural origin - and contain living cells, the required growth factors, as well as nutrition to be processed by an automated biofabrication technology. ${ }^{4,5}$ In most cases direct ink-writing is used, allowing the production of clinically relevant designs with respect to time and size. ${ }^{6}$ Bioinks have to be printable at cell friendly conditions, and allow maturation of the printed construct for several weeks. Several key characteristics can be associated with an ideal hydrogel bioink. First, a pronounced shear thinning character of the precursor hydrogel during extrusion facilitates 3D- 
printing and concurrently the viscoelastic solid like character prevents cell sedimentation in the barrel. $^{7}$ Second, it should ideally allow for sufficient and fast stabilization after the printing process. The former, i.e. the formation of a precursor hydrogel, can be ensured by a wide variety of approaches, such as specific chemical pre-crosslinking ${ }^{8}, \mathrm{pH}^{-9}$ or temperature switch $^{10}$, as described e.g. for alginate, collagen and gelatine. The defined control of these approaches to provide a cytocompatible printing process is an ongoing challenge. Thermogelling polymer solutions that undergo fast gelation are promising candidates as bioinks. A well-known example is the polymer Pluronic F127, also known as Poloxamer 407. This triblock copolymer based on polypropylene glycol thermoresponsive central block flanked by two hydrophilic polyethylene glycol (PEG) blocks forms a physical hydrogel at room temperature. Since Pluronic F127 gels represent excellent printability ${ }^{11}$, it is used in many applications as a support material and sacrificial structure. ${ }^{12}$ One possible alternative for PEG based systems is the family of polymers known as poly(2-oxazoline)s (POx) and their close relative poly(2-substituted-5,6-dihydro-4H1,3-oxazine)s (poly(2-oxazine)s, POzi), which serve as a diverse biomaterials platform for different applications due to good cytocompatibility and chemical versatility. ${ }^{13-17}$ In the context of biofabrication only few reports can be found describing POx/POzi based structures used in bioprinting. Lorson et al. reported a thermogelling diblock copolymer comprising hydrophilic poly(2-methyl-2-oxazoline) (PMeOx) and thermoresponsive poly(2-n-propyl-2-oxazine) ( $\mathrm{P} n \mathrm{PrOzi})$ moieties. In first bioprinting experiments excellent cytocompatibility was confirmed. However, this thermoresponsive hydrogel does not allow for long-term cell culture experiments as it dissolves upon addition of excess cell culture medium. More recently Trachsel et al. investigated enzymatically stabilized hydrophilic poly(2-ethyl-2-oxazoline) hydrogels via sortase linkage. ${ }^{18}$ To use this system in a bioink formulation alginate was needed to stabilize the constructs after printing using $\mathrm{Ca}^{2+}$, and cellulose nanofibrils were added to improve printability. ${ }^{19}$ In recent years, several approaches - mainly based on irradiation with UV-light - have been described to introduce covalent crosslinking after printing, ${ }^{20-22}$ which maintains an 
ongoing controversial discussion on potential negative effect of UV irradiation on cell viability. ${ }^{22,23}$ More recently, crosslinking using visible light has gained attentions. Irrespective of the wavelength used, photoinitiators are typically needed which may affect cells either immediately or during the maturation. ${ }^{22}$ Accordingly, alternative approaches for the crosslinking of hydrophilic polymers to obtain hydrogels remain actively investigated. One such alternative is the Diels-Alder chemistry, already introduced for hydrogel synthesis by Chujo et al. a few decades ago ${ }^{24-26}$ and recently studied again by Shoichet et al. ${ }^{27-30}$ as well as Nahm et al. ${ }^{31}$. Chujo et al. used $\mathrm{PMeOx}$, functionalized with maleimide and furan groups in the polymer side chain. However, these hydrogel precursors would be unlikely candidates for dispense plotting, due to their hydrophilic nature and rheological properties. Here, we translate this strategy to a bioink formulation combining physical and chemical crosslinking, to achieve homogenous cell distribution throughout the construct, good printability, bioinstructive modification and good cytocompatibility.

\section{Results and discussion}

Previously, a diblock copolymer comprising a hydrophilic PMeOx block and a thermoresponsive $\mathrm{P} n \mathrm{PrOzi}$ block $(\mathrm{PMeOx}-b-\mathrm{P} n \mathrm{PrOzi}=\mathbf{P 0})$ has been introduced as bioink ${ }^{32}$ of which the printability and shape fidelity could be significantly improved by addition of Laponite XLG. ${ }^{33}$ However, utilization of the bioink for prolonged studies is hampered by dissolution in long-term cell culture. Therefore, additional chemical crosslinking after printing is necessary, for which we chose the well-known post-polymerization modification approach enabling Diels-Alder chemistry. ${ }^{24,31}$ 


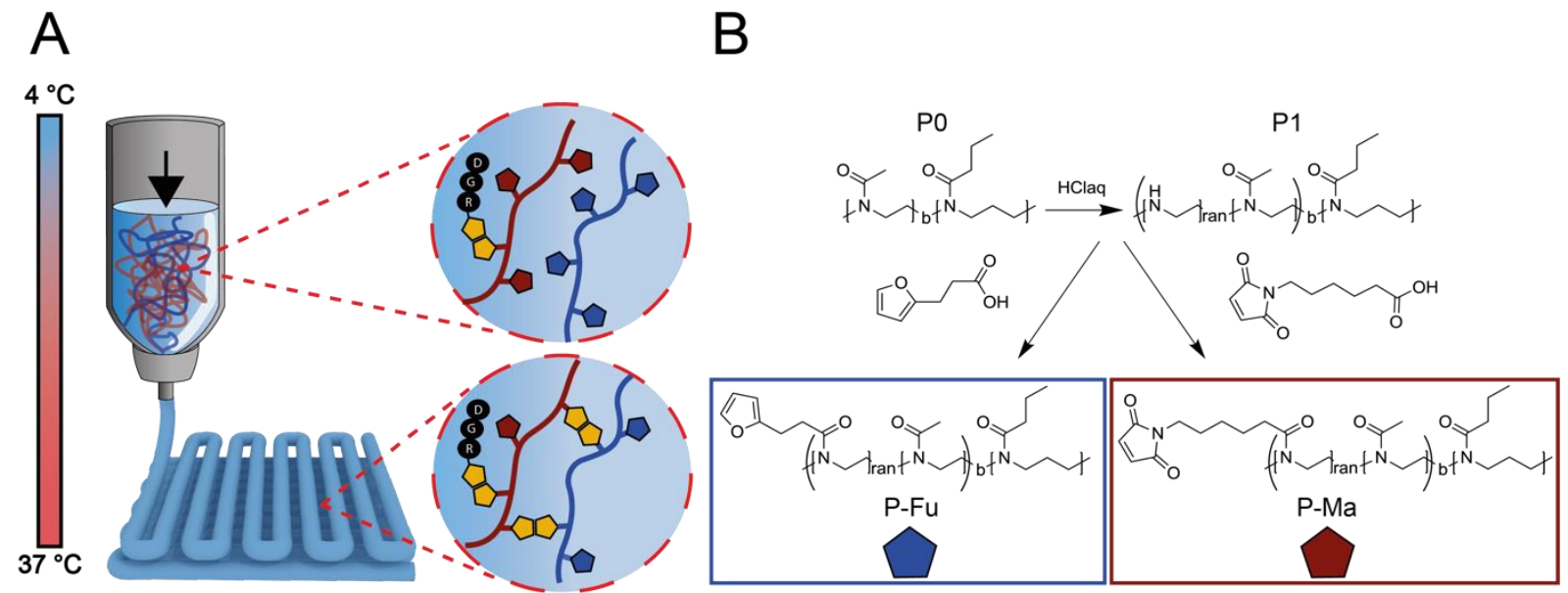

Figure 1/ (A) Schematic illustration of investigated strategy for functional bioink formulations: At $5{ }^{\circ} \mathrm{C}$ the polymer solutions P-Fu and P-Ma present as low viscous liquids, which can be easily mixed with cells and peptide motifs (e.g. RGD). Increasing the temperature to $37{ }^{\circ} \mathrm{C}$ leads to rapid physical gelation of both polymers P-Fu and P-Ma preventing cell sedimentation and ensuring good printability. After printing, the chemical crosslinking takes place at $37{ }^{\circ} \mathrm{C}$ generating stable and biofunctionalized constructs. (B) Synthesis route to establish the thermogelling polymers P-Fu and P-Ma: (A) Partial acidic hydrolysis of the thermogelling diblock copolymer $\mathrm{P} 0\left(\mathrm{PMeOx}_{100}-b-\mathrm{P} n \mathrm{PrOzi}_{100}\right)$ followed by the introduction of furan and maleimide moieties by amide coupling (P-Fu and P-Ma).

Accordingly, our bioink concept builds on two independent crosslinking mechanisms. First, thermoreversible hydrophobic interactions offer excellent handling and printing properties and second, a slow but essentially irreversible Diels-Alder crosslinking post processing for longterm stability and maturation. In addition, Diels-Alder functionalities allow for bioconjugation of bioactive components (Figure 1A). ${ }^{34-36}$ To introduce the corresponding functionalities, the thermogelling diblock copolymer $\mathrm{PMeOx}_{100}-b-\mathrm{P} n \mathrm{PrOzi}_{100}(\mathrm{P} 0)$ was partially hydrolysed yielding secondary amines, which are subsequently coupled with carboxylic acids (Figure 1B). Doing so, it is critical that the thermogelling properties of the polymer P0 (Figure S1) are retained after modification and that the crosslinking occurs in a time period suitable for bioprinting. The first step was a carefully controlled partial hydrolysis of the polymer. From literature, we expected that the $\mathrm{MeOx}$ units are hydrolysed significantly faster than the $n$ PrOzi units. ${ }^{37,38}{ }^{1} \mathrm{H}-$ NMR spectroscopy confirmed that backbone and sidechain signal attributed to MeOx units decreased significantly with increasing reaction time (Figure S2), while the PnPrOzi signals 
remained preserved. Here, $10 \%$ of the $\mathrm{PMeOx}$ block was hydrolyzed yielding ethyleneimine (EI) moieties in the hydrophilic part of the polymer $\left(\left(\mathrm{P}\left(\mathrm{MeOx}_{90}-c o-\mathrm{EI}_{10}\right)-b-\mathrm{P} n \mathrm{PrOzi}_{100}=\mathrm{P} 1\right)\right.$ (Figure S3). The thermogelling properties of 3 different P1 polymer batches performed similar like the unmodified polymers described by Lorson et. al (Table S1) ${ }^{32}$, indicating the reproducibility of the approach. ${ }^{39}$ Modification of $\mathrm{P} 1$ with furan- or maleimide moieties resulted to the final functionalized polymers $\mathrm{P}-\mathrm{Fu}\left(\mathrm{P}\left(\mathrm{MeOx}_{90}-c o-\mathrm{Fu}_{10}\right)-b-\mathrm{P} n \mathrm{PrOzi}{ }_{100}\right)$ and $\mathrm{P}-\mathrm{Ma}$ ( $\left.\mathrm{P}\left(\mathrm{MeO}_{90}-c o-\mathrm{Ma}_{10}\right)-\mathrm{b}-\mathrm{P} n \mathrm{PrOzi}_{100}\right)$. The successful and complete modification was verified by ${ }^{1} \mathrm{H}-\mathrm{NMR}$ experiments (Figure S3) and all relevant singals could be attributed. Important to note, the signals attributed to EI units completely disappeared. Furthermore, complete modification of all secondary amines was confirmed via titration (Figure S3).

All derivatives of $\mathrm{P} 0$ exhibited pronounced thermogelling properties in the temperature range of $5{ }^{\circ} \mathrm{C}-40{ }^{\circ} \mathrm{C}\left(5^{\circ} \mathrm{C}\right.$ low viscous liquid, $40{ }^{\circ} \mathrm{C}$ stable hydrogel, Figure 2$)$ with sol/gel transitions between 26 and $29{ }^{\circ} \mathrm{C}$ (Figure 2). Compared to the precursor polymer P0 the transition temperature increased, rendering handling at room temperature easier $\left(\mathrm{T}_{\text {gel }}(\mathrm{P} 0)_{-}=21^{\circ} \mathrm{C}\right.$, Figure S1). Notably, the storage modulus at $37{ }^{\circ} \mathrm{C}$ increased from $3.8 \mathrm{kPa}$ for $\mathrm{P} 0$ to $5.3 \mathrm{kPa}$ for P1 (Figure 2A). In contrast, the addition of furan and maleimide moieties resulted to a reversal of storage modulus to $3.3 \mathrm{kPa}$ and $3.0 \mathrm{kPa}$, respectively (Figure $2 \mathrm{~B}, \mathrm{C}$ ). Clearly, modifications of the hydrophilic block affects the polymer self-assembly, presumably by affecting the compatibility between the blocks. The physical hydrogels $\mathrm{P}-\mathrm{Fu}$ and $\mathrm{P}-\mathrm{Ma}$ at $37{ }^{\circ} \mathrm{C}$ and a concentration of $20 \mathrm{wt} . \%$ were further characterized individually via oscillatory and rotational shear rheology to investigate whether their rheological parameters would be favourable for 3D printing (Figure 3). 

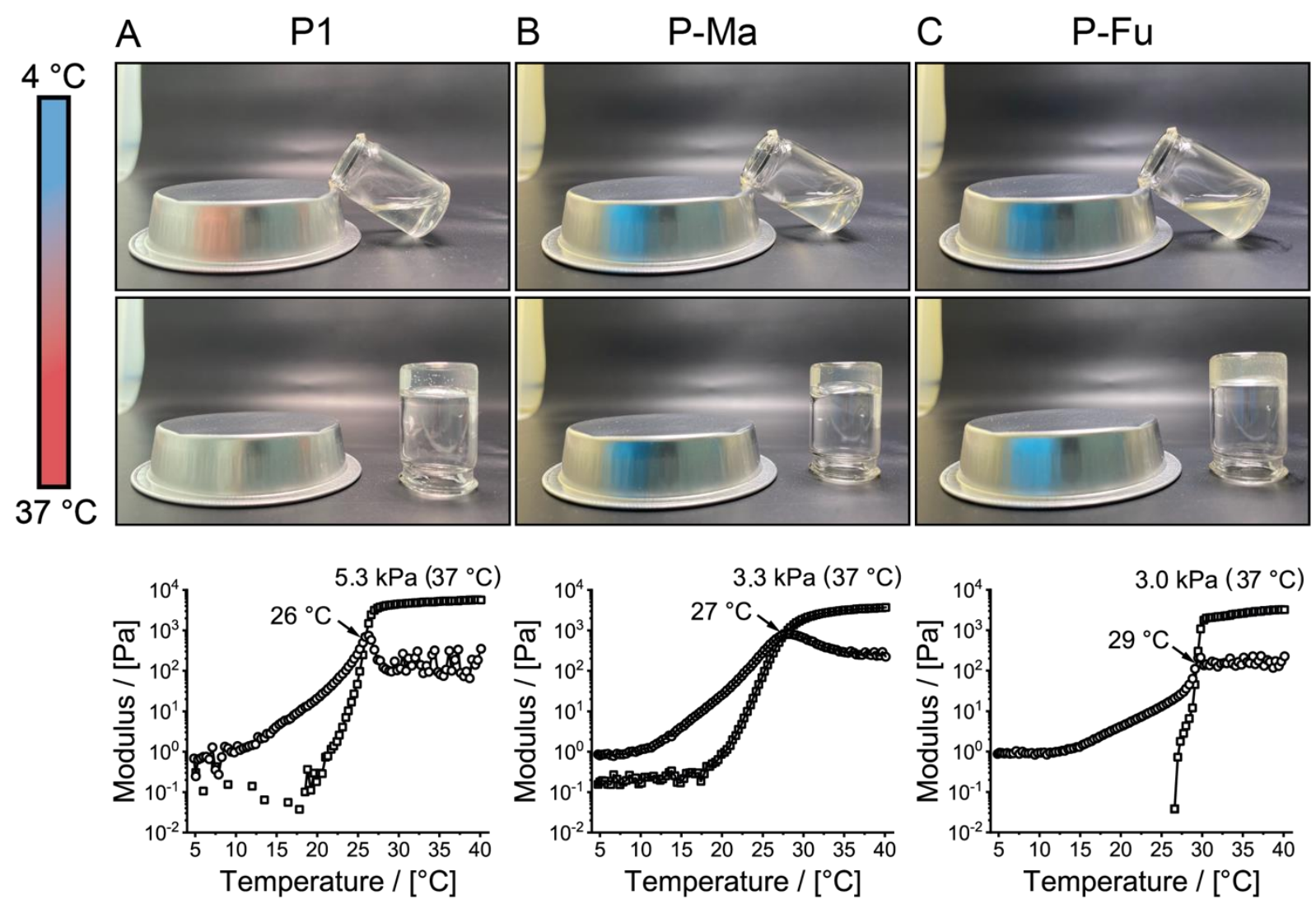

Figure 2| Thermogelling properties $\mathrm{P} 1(\mathrm{~A}), \mathrm{P}-\mathrm{Ma}(\mathrm{B})$ and $\mathrm{P}-\mathrm{Fu}(\mathrm{C})$ in the temperature range of 5 to $37^{\circ} \mathrm{C}$ (heat rate: $0.05^{\circ} \mathrm{C} / \mathrm{s}$ ) at $20 \mathrm{wt} . \%$ aqueous solutions ( $\square$ : Storage modulus $\mathrm{G}^{\prime}, \circ$ : Loss modulus $\mathrm{G}^{\prime \prime}$ ). Images where taken at $5{ }^{\circ} \mathrm{C}$ and $37^{\circ} \mathrm{C}$ following the described temperature scale.

Both samples showed a pronounced linear viscoelastic region in the amplitude sweep (Figure 3B). Slightly higher $\mathrm{G}^{\prime}$ values are obtained for the polymer P-Ma, which is in agreement with the values obtained during the temperature sweep (Figure 2). In the investigated frequency region both polymers exhibited viscoelastic solid-like character throughout (Figure 3C). The pronounced shear-thinning (Figure $3 \mathrm{D}$ ) with flow indices of $\mathrm{n}=0.15$ for P-Fu and P-Ma, welldefined yield-points (Figure $3 \mathrm{E} ; \tau(\mathrm{P}-\mathrm{Fu})=92 \mathrm{~Pa}$ and $\tau(\mathrm{P}-\mathrm{Ma})=166 \mathrm{~Pa})$, high viscosity at low shear stress (ca. $100 \mathrm{kPa} * \mathrm{~s}$ ) and fast structure recovery (Figure 3F) suggests good printability for both bioinks. This rheological profile allows the low viscosity polymers sols to be mixed with cells at $\leq 10{ }^{\circ} \mathrm{C}$, and subsequently printed at $37^{\circ} \mathrm{C}$ on a preheated printing dish, where the crosslinking Diels/Alder reaction takes place subsequently (Figure 4A). 

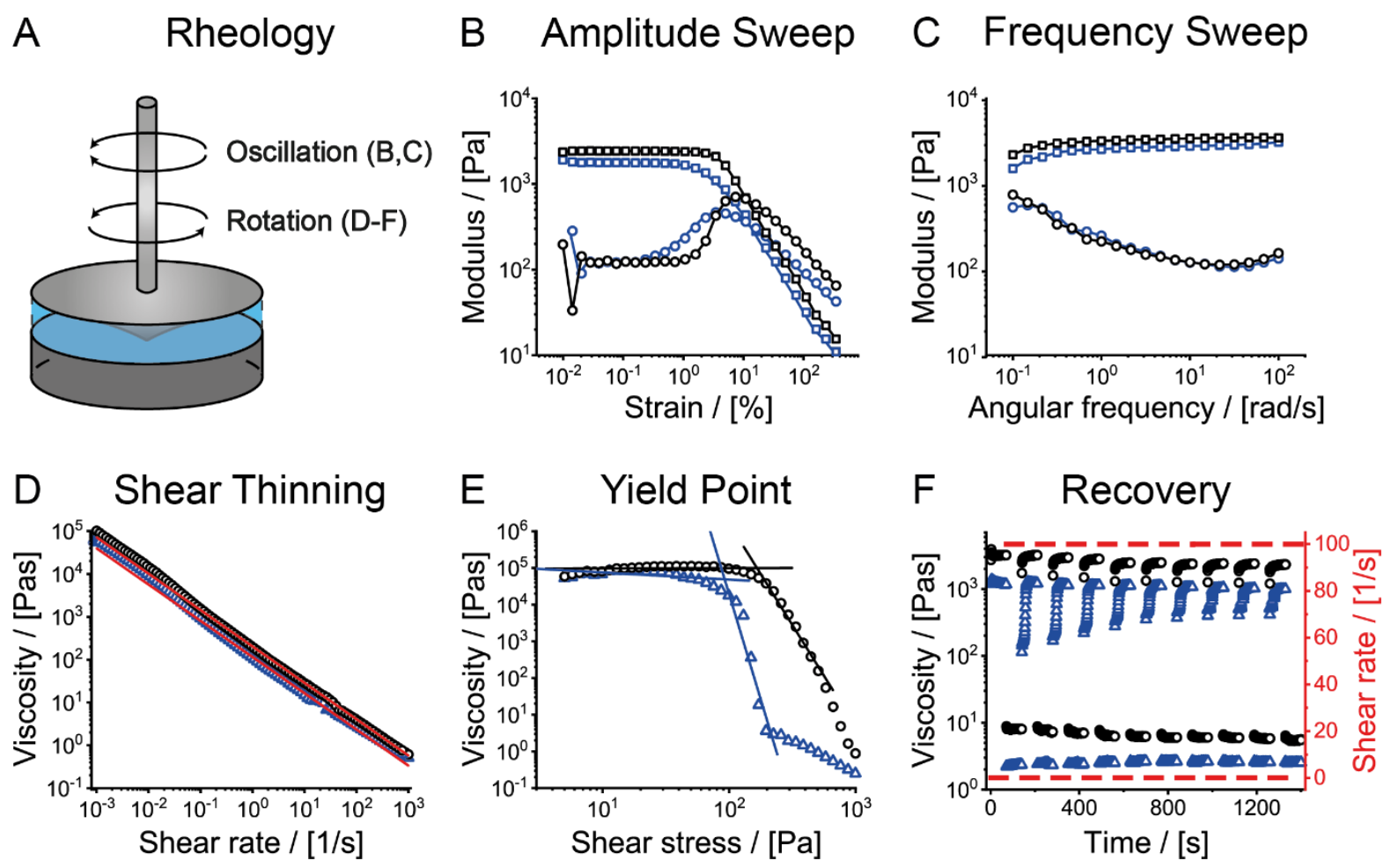

Figure 3| Determination of important rheological properties (A) for direct ink-writing of P-Fu (blue) and P-Ma (black) at a concentration of $20 \mathrm{wt.} \%$ and $37^{\circ} \mathrm{C}$. (B) Amplitude sweep (口: Storage modulus $\mathrm{G}^{\prime}, \mathrm{O}$ : Loss modulus $\mathrm{G}^{\prime \prime}$ ) and frequency sweep (C). D) Shear thinning properties: Viscosity in dependence of the applied shear rate. Red line: data fitted with a power law function. E) Yield point determination: Viscosity as a function of applied shear stress. The onset of viscosity decrease designates the yield point $\tau$. F) Structure-recovery properties: Alternated high and low-shear regimes.

Accordingly, we mixed $20 \mathrm{wt} . \%$ aqueous solutions of both polymers $(1 / 1, \mathrm{v} / \mathrm{v})$ at $10{ }^{\circ} \mathrm{C}$ and followed $\mathrm{G}^{\prime}$ and $\mathrm{G}^{\prime}$ ' at $10{ }^{\circ} \mathrm{C}, 20^{\circ} \mathrm{C}$ and $37{ }^{\circ} \mathrm{C}$ over time (Figure 4B). Over the investigated time of $2 \mathrm{~h}$ no hydrogel formation was observed at $10{ }^{\circ} \mathrm{C}$ and the sample remained a low viscous liquid, ideal for sample preparation, cell distribution and transfer into a printing syringe. Only a minor increase in viscosity is observed after approx. 1h. With a temperature-controlled printing setup, this allows for prolonged printability, if needed. In contrast, at $25^{\circ} \mathrm{C}$, which is below $\mathrm{T}_{\text {gel }}$ hydrogel, a sol/gel transition is observed after 65 minutes and must be attributed to the Diels-Alder crosslinking. In contrast, at $37^{\circ} \mathrm{C}$ the mixture thermogelled immediately, followed by the Diels/Alder crosslinking, as evidenced by an increase of G' and G''. After less than 50 minutes a plateau value of more than $10 \mathrm{kPa}$ was reached. Accordingly, printing the 
bioink at room temperature onto a preheated dish should ensure rapid crosslinking and stability of the printed construct.
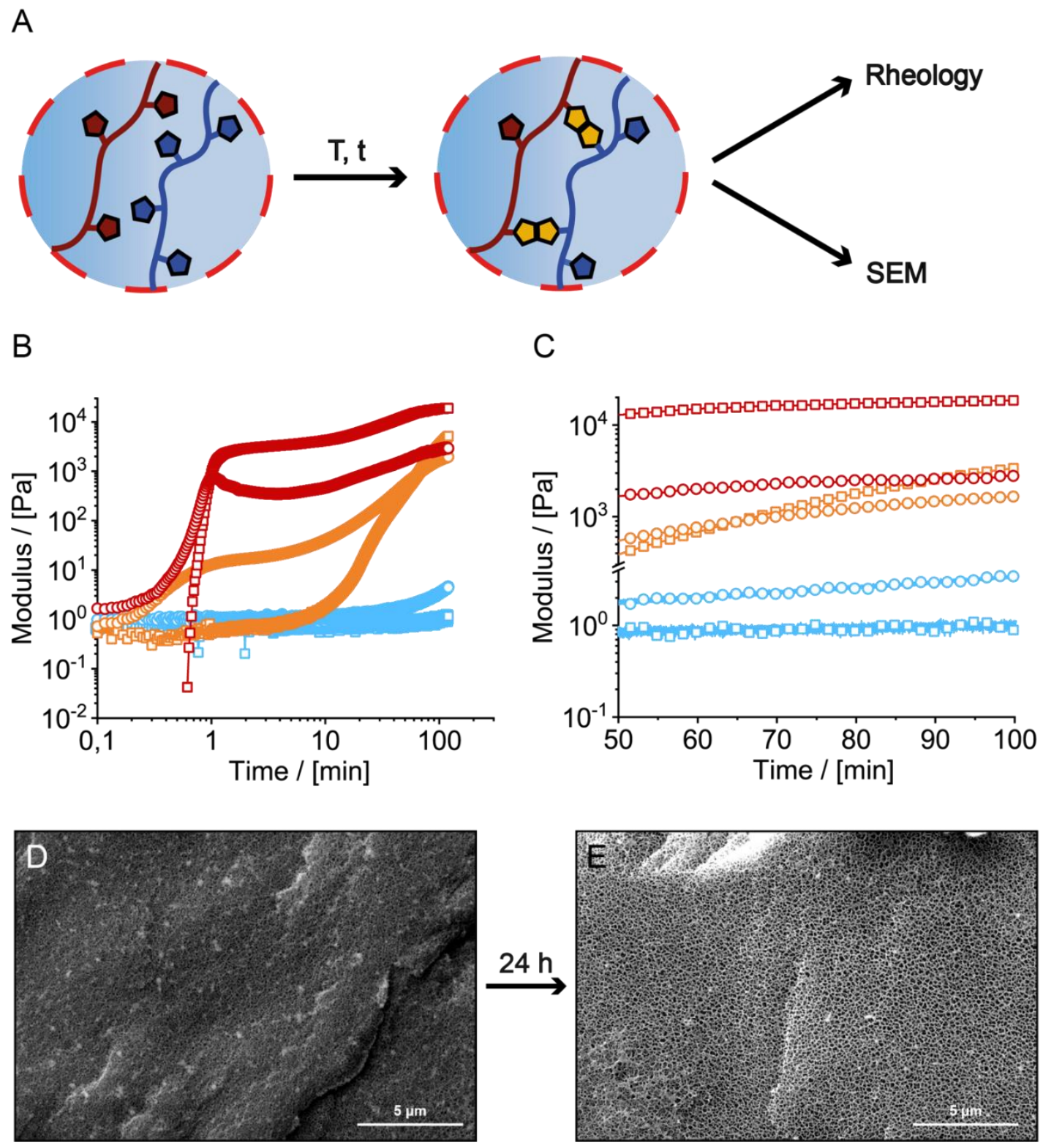

Figure 4| Chemical crosslinking of mixed P-Fu and P-Ma hydrogels (A) Workflow for crosslinking analysis (B-C) Crosslinking kinetics of P-Fu and P-Ma mixtures (1:1) at different temperatures (blue: $10{ }^{\circ} \mathrm{C}$, orange: $20{ }^{\circ} \mathrm{C}$ and red: $37{ }^{\circ} \mathrm{C}$ ) and 20 wt.\% concentration ( $\square$ : Storage modulus $\mathrm{G}^{\prime}, \circ$ : Loss modulus $\mathrm{G}^{\prime \prime}$ ): Time scans of $120 \mathrm{~min}(\mathrm{~B})$ with a fixed amplitude of $0.5 \%$ and an angular frequency of $10 \mathrm{rad} / \mathrm{s}$ with a (C) detailed view for 50-100 min which we deem critical for preparing and conducting a typical print. (D-E) Cryogenic scanning electron microscopy investigations to visualize the porous structure of the bioink: Samples were recorded after crosslinking of $24 \mathrm{~h}$ (D) and additional swelling for $24 \mathrm{~h}(\mathrm{E})$. 
Due to the nature of the chemical crosslinked synthetic hydrogel, a highly porous network with features in the range of a few dozen nm was obtained (Figure 4D). Swelling for $24 \mathrm{~h}$ led to a significant increase of pore size into the lower 100-nm range (Figure 4 E). Although the pore size is sufficiently large for diffusion of nutrients, cells will not be able to migrate through the generated network. Compared to the physical hydrogel of P0 (Figure S1 B) the pore size decreased significantly after chemical crosslinking due to the formation of a more compact three-dimensional covalent network. In order to highlight the adaptability of the platform in terms of stiffness the concentration of precursor solutions of P-Fu and P-Ma was decreased by dilution with water leading to softer hydrogels with slower crosslinking kinetics (Figure S4). Additionally, the nature of the crosslinker can be adapted to specific applications. To demonstrate this, we used $\mathrm{PEG}_{600}$-bismaleimide as a model crosslinker. The P-Fu-PEG mixtures showed a slower crosslinking and resulted in softer and less dense network as characterized by rheology and cryoSEM, respectively (Figure S5). Not surprising, this led to a more pronounced swelling of the hydrogels compared to the P-Fu/P-Ma crosslinking.

Based on the favourable rheological properties of the precursor hydrogels and the controlled crosslinking kinetics, both printability and long-term stability were further analysed. At $5{ }^{\circ} \mathrm{C}$ P-Fu and P-Ma solutions were homogenously mixed and transferred into a printing cartridge at (held at $5{ }^{\circ} \mathrm{C}$ ) followed by printing onto a preheated $\left(37^{\circ} \mathrm{C}\right)$ printing bed (Figure 5 A-C). After 1 hour of incubation at $37{ }^{\circ} \mathrm{C}$ the constructs were immersed in fresh cell culture medium and incubated for 14 days at $37^{\circ} \mathrm{C}$. The constructs remained stable with good shape fidelity (Figure 
5 D). Even though the hydrogels are soft as analyzed by rheology as shown above they were easily handled and transferred while retaining their printed shape. (Figure 5 D - G).
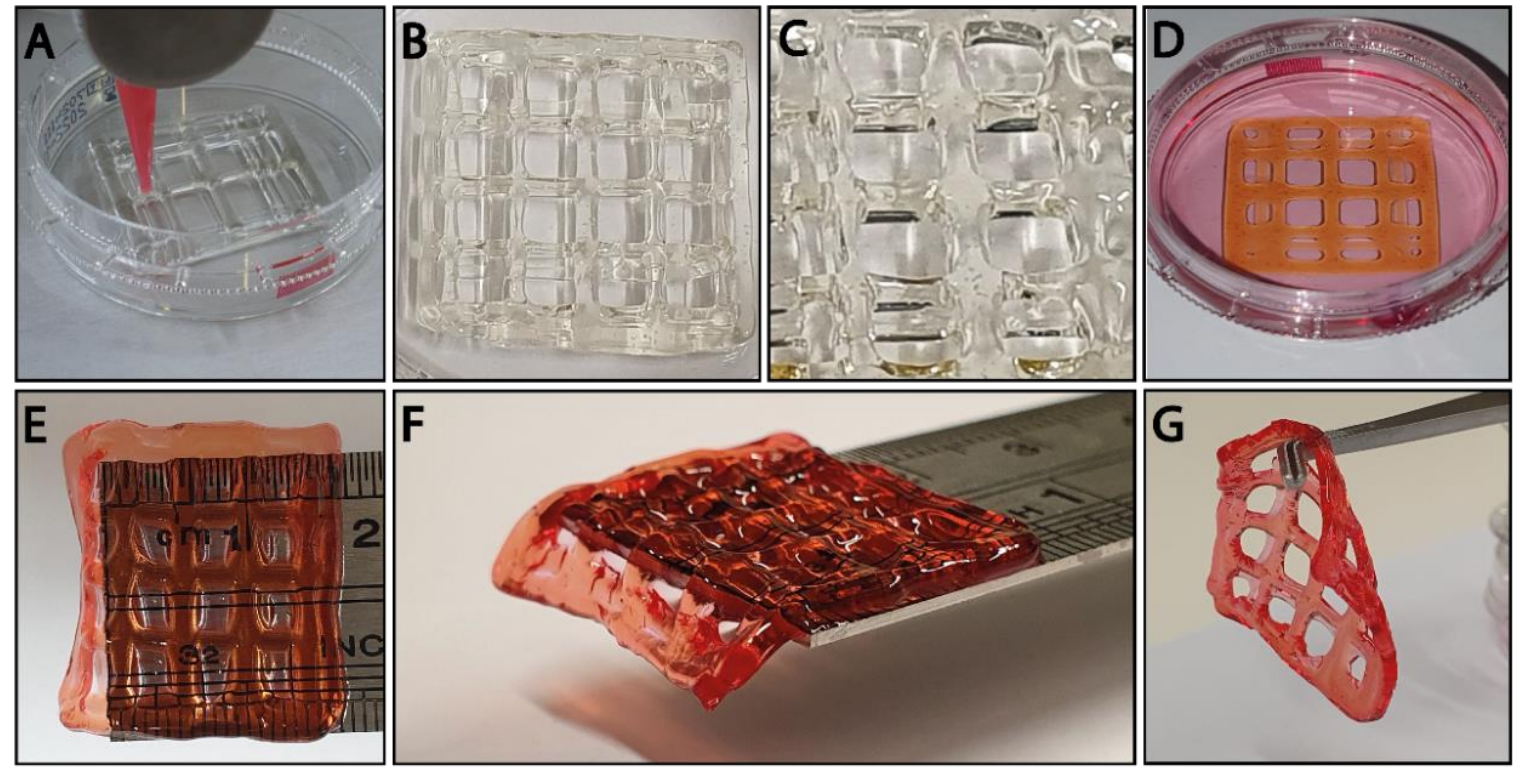

Figure 5| Printing of FuP and MaP crosslinking hydrogels: (A) Image of the printed scaffold during the printing process. (B-C) Illustration of the printed scaffold immediately after printing. (D) Printed scaffolds after incubation in culture medium for 14 days. (E-G) Handling and transfer of FuP and MaP crosslinked hydrogels after 14 days in culture medium.

As mentioned above, it has been shown that different POx-based hydrogels show no cytotoxic effects on cells and therefore form a promising platform for bioinks. ${ }^{40-44}$ However, applicability in biomedicine requires the adhesive functionalization of POx based hydrogels, as the material per se does not support cell adhesion. To test, whether adhesive functionalization of our hydrogels supports cell adhesion, we functionalized them with integrin-ligating RGD-peptide. We further generated lentivirally transduced, NIH/3T3-based morphology reporter cells stably expressing farnesylated tdTomato red-fluorescent protein to label the plasma membrane, and compared the morphology of our reporter cells cultured on top of RGD-functionalized and nonfunctionalized POx hydrogels. Epifluorescence microscopy analyses after 4 and 5 days of cultivation revealed that cells on non-functionalized POx hydrogels were low in number and showed a rounded morphology (Fig. 6A), whereas cells on RGD-functionalized hydrogels displayed a well spread fibroblastoid morphology (Fig. 6B) and even increased in number over 
time (Fig. 6C). These data demonstrate that adhesive functionalization of our POx hydrogels supports cell adhesion, spreading and proliferation.
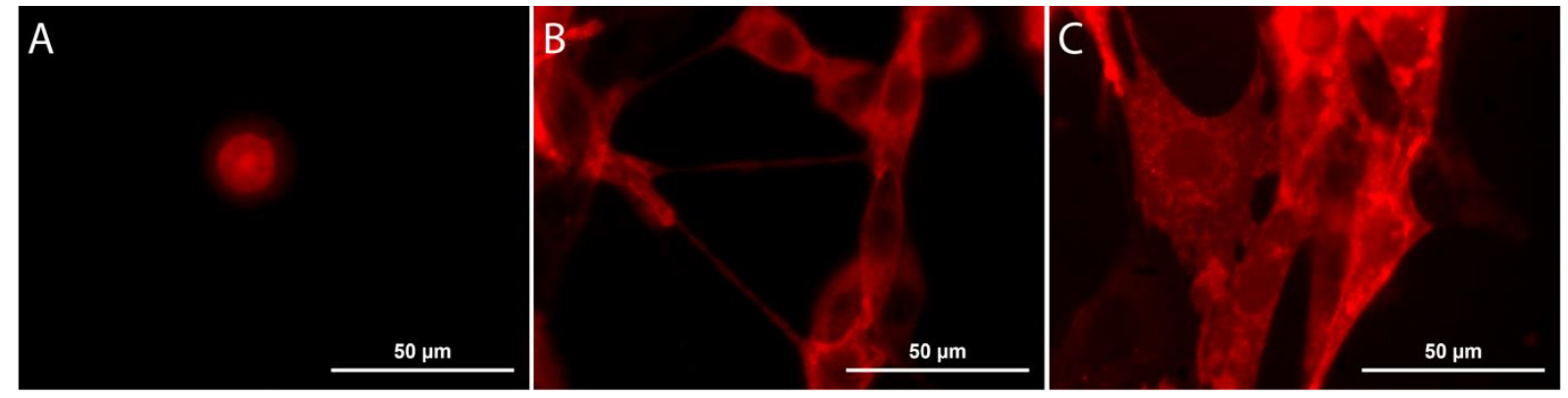

Figure $6 \mid$ Cell adhesion on the surface of P-Fu and P-Ma crosslinking hydrogels. (A) POx hydrogel without RGD-modification does not allow for good cell adhesion and spreading. In contrast, cell adhesion and spreading was observed on POx hydrogel with RGD-modification after (B) 4 days of and (C) 5 days of cultivation.

To further study cell viability within the gel (3D), NIH3T3 cells were embedded in the hydrogel pre-printing. After the printing process and cross-linking of the hydrogels the cell viability of the encapsulated cells in the scaffold was analysed. Cells were pre-stained with Hoechst 33342 prior to the printing process. This was necessary as preliminary work showed that FDA was not able to penetrate the gels in a sufficient manner. Dead cells were visualized using PI (here no incompatibilities with in-gel penetration where observed) staining after 1 and 2 days. After optimizing the polymer purification process (Fig. S6) as well as the printing protocol cell viability staining showed no cytotoxic effect of the hydrogel on the cells. No difference between the unmodified and RGD-functionalized constructs was observed. The majority of dead cells that were visible scanning the entire printed hydrogel construct could be accounted to dryingoff effects at the outer layers, which was reported for other hydrogel systems before (Figure S7). ${ }^{45,46}$ Despite the RGD-functionalization the cells showed a rounded morphology after the encapsulation in the hydrogel (Figure 7). It has been previously described that POx hydrogels with moduli of around of around $3.5-4.5 \mathrm{kPa}$ are too stiff for the migration of the cells. ${ }^{47,48}$ Furthermore, small pore sizes as described above for this system have been shown to prevent cell spreading and migration. ${ }^{49,50}$ With a storage modulus of around $10 \mathrm{kPa}$ and submicron pore 
size, the hydrogel system as shown in this study clearly does not allow the migration and therefore also the spreading of the cells.

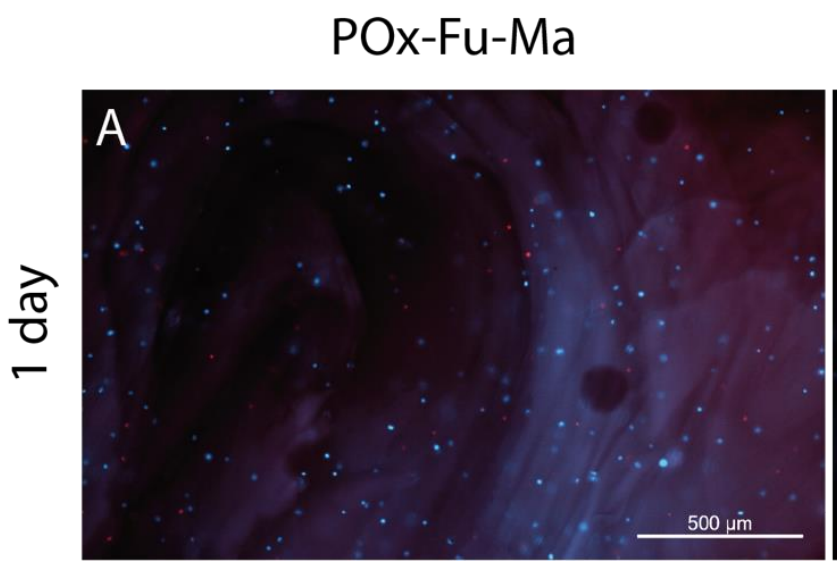

POx-Fu-Ma + RGD
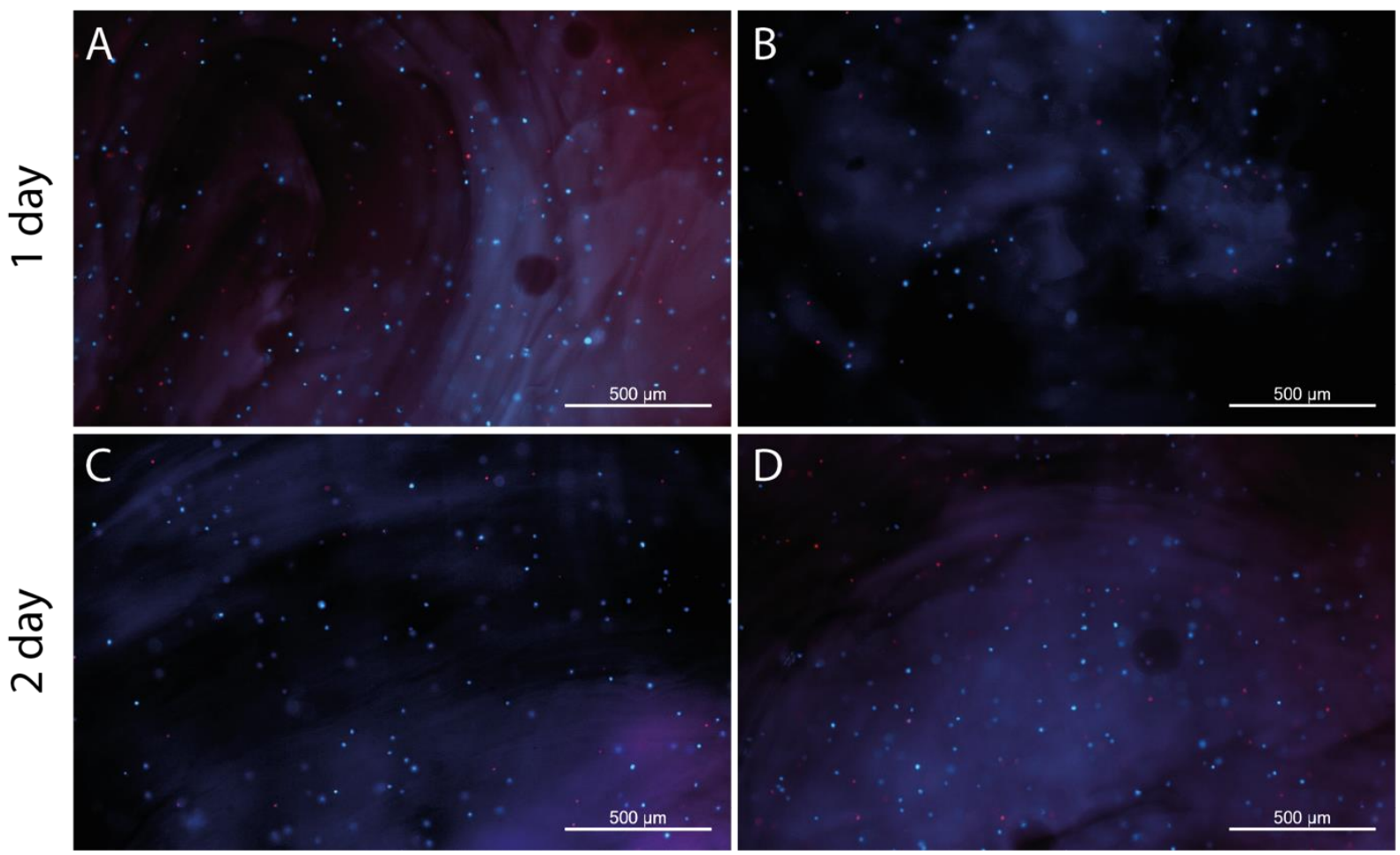

Figure 7| Bioprinting of fibroblasts in P-Fu/P-Ma-bioinks. NIH3T3 cells were pre-stained with Hoechst 33342 (blue) and incorporated, printed and cultivated in POx-based hydrogels (20 wt.\%) with (B and D) and without RGD-peptide (A and C). Dead cells were assessed by staining with propidium iodide (red).

In an effort to address cell adhesion and migration we aim at incorporating matrix metalloprotease (MMP) cleavable linkers into the hydrogel network to ensure cleavage by secreted MMP and therefore a loosening of the network as demonstrated before. ${ }^{51-53}$ The sequence of the Linker (GPQGIAGQ) is derived from collagen. It has been shown to be responsive to MMP cleavage and has already been used in different applications. ${ }^{54-57}$ The Linker can be flanked with either Thiol or maleimide groups for the cross-linking of both P-Fu and P-Ma and is part of the ongoing research. 


\section{Conclusion}

In this study, favourable thermogelation and shear-thinning properties were combined with cell friendly post printing chemical crosslinking via Diels/Alder chemistry. The postpolymerization modification preserved the nature of physical crosslinked hydrogels. The crosslinking kinetics and density could be fine-tuned with different temperatures and crosslinking degrees. The second crosslinking step ensured stability of printed constructs over several weeks. Biofunctionality was introduced via the attachment of RGD binding motives and NIH 3T3 cells showed cell adhesion and an elongated morphology when seeded on top of the hydrogels for a couple of days. First bioprinting experiments highlighted the optimized printing setup, the biocompatibility and the functionality of the investigated bioink formulation.

\section{Acknowledgement}

The authors would like to gratefully acknowledge support by the Deutsche Forschungsgemeinschaft (DFG, German Research Foundation)-project number 326998133TRR225 (subprojects A03, B06). Furthermore, we thank the Deutsche Forschungsgemeinschaft for funding the crossbeam scanning electron microscope Zeiss CB 340 (INST 105022/58-1 FUGG) within the DFG State Major Instrumentation Programme.

\section{Supporting Information}

Detailed description of material and methods including synthesis and characterization of investigated polymer structures. Kinetic investigation of acidic hydrolysis. The influence of hydrolysis degree on thermogelling properties. Chemical crosslinking of P-Fu and P-Ma at different concentrations and ratios. Chemical crosslinking via PEG-bismaleimide structures (Rheology, cryoSEM, stability after printing). 


\section{References}

1. Moroni, L.; Boland, T.; Burdick, J. A.; De Maria, C.; Derby, B.; Forgacs, G.; Groll, J.; Li, Q.; Malda, J.; Mironov, V. A.; Mota, C.; Nakamura, M.; Shu, W.; Takeuchi, S.; Woodfield, T. B. F.; Xu, T.; Yoo, J. J.; Vozzi, G., Biofabrication: A Guide to Technology and Terminology. Trends in biotechnology 2018, 36 (4), 384-402.

2. Chimene, D.; Lennox, K. K.; Kaunas, R. R.; Gaharwar, A. K., Advanced Bioinks for 3D Printing: A Materials Science Perspective. Annals of biomedical engineering 2016, 44 (6), 2090-2102.

3. Valot, L.; Martinez, J.; Mehdi, A.; Subra, G., Chemical insights into bioinks for 3D printing. Chemical Society Reviews 2019, 48 (15), 4049-4086.

4. Groll, J.; Burdick, J. A.; Cho, D. W.; Derby, B.; Gelinsky, M.; Heilshorn, S. C.; Jüngst, T.; Malda, J.; Mironov, V. A.; Nakayama, K.; Ovsianikov, A.; Sun, W.; Takeuchi, S.; Yoo, J. J.; Woodfield, T. B. F., A definition of bioinks and their distinction from biomaterial inks. Biofabrication 2018, 11 (1), 013001.

5. Groll, J.; et al., Biofabrication: reappraising the definition of an evolving field. Biofabrication 2016, 8 (1), 13001.

6. Chimene, D.; Kaunas, R.; Gaharwar, A. K., Hydrogel Bioink Reinforcement for Additive Manufacturing: A Focused Review of Emerging Strategies. 2020, 32 (1), 1902026.

7. Jungst T, S. W. S. K. S. T.; Groll, J., Strategies and molecular design criteria for 3D printable hydrogels. Chem. Rev. 2016, 116, 1496.

8. Hazur, J.; Detsch, R.; Karakaya, E.; Kaschta, J.; Teßmar, J.; Schneidereit, D.; Friedrich, O.; Schubert, D. W.; Boccaccini, A. R., Improving alginate printability for biofabrication: establishment of a universal and homogeneous pre-crosslinking technique. Biofabrication 2020, 12 (4), 045004.

9. Osidak, E. O.; Kozhukhov, V. I.; Osidak, M. S.; Domogatsky, S. P., Collagen as Bioink for Bioprinting: A Comprehensive Review. Int J Bioprint 2020, 6 (3), 270-270.

10. Ouyang, L.; Yao, R.; Zhao, Y.; Sun, W., Effect of bioink properties on printability and cell viability for 3D bioplotting of embryonic stem cells. Biofabrication 2016, 8 (3), 035020.

11. Müller, M.; Becher, J.; Schnabelrauch, M.; Zenobi-Wong, M., Nanostructured Pluronic hydrogels as bioinks for 3D bioprinting. Biofabrication 2015, 7 (3), 035006.

12. Kolesky, D. B.; Truby, R. L.; Gladman, A. S.; Busbee, T. A.; Homan, K. A.; Lewis, J. A., 3D Bioprinting of Vascularized, Heterogeneous Cell-Laden Tissue Constructs. 2014, 26 (19), 3124-3130.

13. Lorson, T.; Lübtow, M. M.; Wegener, E.; Haider, M. S.; Borova, S.; Nahm, D.; Jordan, R.; Sokolski-Papkov, M.; Kabanov, A. V.; Luxenhofer, R., Poly(2-oxazoline)s based biomaterials: A comprehensive and critical update. Biomaterials 2018, 178, 204-280.

14. Park, J.-R.; Bolle, E. C. L.; Santos Cavalcanti, A. D.; Podevyn, A.; Van Guyse, J. F. R.; Forget, A.; Hoogenboom, R.; Dargaville, T. R., Injectable biocompatible poly(2-oxazoline) hydrogels by strain promoted alkyne-azide cycloaddition. Biointerphases 2021, 16 (1), 011001. 15. Lübtow, M. M.; Lorson, T.; Finger, T.; Gröber-Becker, F.-K.; Luxenhofer, R., Combining Ultra-High Drug-Loaded Micelles and Injectable Hydrogel Drug Depots for Prolonged Drug Release. Macromolecular Chemistry and Physics 2020, 221 (1), 1900341.

16. Lübtow, M. M.; Oerter, S.; Quader, S.; Jeanclos, E.; Cubukova, A.; Krafft, M.; Haider, M. S.; Schulte, C.; Meier, L.; Rist, M.; Sampetrean, O.; Kinoh, H.; Gohla, A.; Kataoka, K.; Appelt-Menzel, A.; Luxenhofer, R., In Vitro Blood-Brain Barrier Permeability and Cytotoxicity of an Atorvastatin-Loaded Nanoformulation Against Glioblastoma in 2D and 3D Models. Molecular Pharmaceutics 2020, 17 (6), 1835-1847.

17. Humphries, J.; Pizzi, D.; Sonderegger, S. E.; Fletcher, N. L.; Houston, Z. H.; Bell, C. A.; Kempe, K.; Thurecht, K. J., Hyperbranched Poly(2-oxazoline)s and Poly(ethylene glycol): A Structure-Activity Comparison of Biodistribution. Biomacromolecules 2020, 21 (8), 3318-3331. 
18. Trachsel, L.; Broguiere, N.; Rosenboom, J.-G.; Zenobi-Wong, M.; Benetti, E. M., Enzymatically crosslinked poly(2-alkyl-2-oxazoline) networks for 3D cell culture. Journal of Materials Chemistry B 2018.

19. Trachsel, L.; Johnbosco, C.; Lang, T.; Benetti, E. M.; Zenobi-Wong, M., DoubleNetwork Hydrogels Including Enzymatically Crosslinked Poly-(2-alkyl-2-oxazoline)s for 3D Bioprinting of Cartilage-Engineering Constructs. Biomacromolecules 2019.

20. Stichler, S.; Jungst, T.; Schamel, M.; Zilkowski, I.; Kuhlmann, M.; Bock, T.; Blunk, T.; Tessmar, J.; Groll, J., Thiol-ene Clickable Poly(glycidol) Hydrogels for Biofabrication. Annals of biomedical engineering 2017, 45 (1), 273-285.

21. Bertlein, S.; Brown, G.; Lim, K. S.; Jungst, T.; Boeck, T.; Blunk, T.; Tessmar, J.; Hooper, G. J.; Woodfield, T. B. F.; Groll, J., Thiol-Ene Clickable Gelatin: A Platform Bioink for Multiple 3D Biofabrication Technologies. 2017, 29 (44), 1703404.

22. Lim, K. S.; Schon, B. S.; Mekhileri, N. V.; Brown, G. C. J.; Chia, C. M.; Prabakar, S.; Hooper, G. J.; Woodfield, T. B. F., New Visible-Light Photoinitiating System for Improved Print Fidelity in Gelatin-Based Bioinks. ACS Biomaterials Science \& Engineering 2016, 2 (10), 1752-1762.

23. Han, W. T.; Jang, T.; Chen, S.; Chong, L. S. H.; Jung, H.-D.; Song, J., Improved cell viability for large-scale biofabrication with photo-crosslinkable hydrogel systems through a dual-photoinitiator approach. Biomaterials Science 2020, 8 (1), 450-461.

24. Chujo, Y.; Sada, K.; Saegusa, T., Reversible gelation of polyoxazoline by means of Diels-Alder reaction. Macromolecules 1990, 23 (10), 2636-2641.

25. Chujo, Y.; Sada, K.; Saegusa, T., Polyoxazoline having a coumarin moiety as a pendant group. Synthesis and photogelation. Macromolecules 1990, 23 (10), 2693-2697.

26. Chujo, Y.; Sada, K.; Saegusa, T., Synthesis of Bipyridyl-Branched Polyoxazoline and Its Gelation by Means of Metal Coordination. Polymer Journal 1993, 25 (6), 599-608.

27. Nimmo, C. M.; Owen, S. C.; Shoichet, M. S., Diels-Alder Click Cross-Linked Hyaluronic Acid Hydrogels for Tissue Engineering. Biomacromolecules 2011, 12 (3), 824-830.

28. Baker, A. E. G.; Tam, R. Y.; Shoichet, M. S., Independently Tuning the Biochemical and Mechanical Properties of 3D Hyaluronan-Based Hydrogels with Oxime and Diels-Alder Chemistry to Culture Breast Cancer Spheroids. Biomacromolecules 2017, 18 (12), 4373-4384. 29. Smith, L. J.; Taimoory, S. M.; Tam, R. Y.; Baker, A. E. G.; Binth Mohammad, N.; Trant, J. F.; Shoichet, M. S., Diels-Alder Click-Cross-Linked Hydrogels with Increased Reactivity Enable 3D Cell Encapsulation. Biomacromolecules 2018, 19 (3), 926-935.

30. Delplace, V.; Nickerson, P. E. B.; Ortin-Martinez, A.; Baker, A. E. G.; Wallace, V. A.; Shoichet, M. S., Nonswelling, Ultralow Content Inverse Electron-Demand Diels-Alder Hyaluronan Hydrogels with Tunable Gelation Time: Synthesis and In Vitro Evaluation. Advanced Functional Materials 2020, 30 (14), 1903978.

31. Nahm, D.; Weigl, F.; Schaefer, N.; Sancho, A.; Frank, A.; Groll, J.; Villmann, C.; Schmidt, H.-W.; Dalton, P. D.; Luxenhofer, R., A versatile biomaterial ink platform for the melt electrowriting of chemically-crosslinked hydrogels. Materials Horizons 2020, 7 (3), 928933.

32. Lorson, T.; Jaksch, S.; Lubtow, M. M.; Jungst, T.; Groll, J.; Luhmann, T.; Luxenhofer, R., A Thermogelling Supramolecular Hydrogel with Sponge-Like Morphology as a Cytocompatible Bioink. Biomacromolecules 2017, 18 (7), 2161-2171.

33. Hu, C.; Hahn, L.; Yang, M.; Altmann, A.; Stahlhut, P.; Groll, J.; Luxenhofer, R., Improving printability of a thermoresponsive hydrogel biomaterial ink by nanoclay addition. Journal of Materials Science 2021, 56 (1), 691-705.

34. Braun, A. C.; Gutmann, M.; Lühmann, T.; Meinel, L., Bioorthogonal strategies for site-directed decoration of biomaterials with therapeutic proteins. J Control Release 2018, 273, 68-85. 
35. St. Amant, A. H.; Lemen, D.; Florinas, S.; Mao, S.; Fazenbaker, C.; Zhong, H.; Wu, H.; Gao, C.; Christie, R. J.; Read de Alaniz, J., Tuning the Diels-Alder Reaction for Bioconjugation to Maleimide Drug-Linkers. Bioconjugate Chemistry 2018, 29 (7), 2406-2414. 36. St. Amant, A. H.; Huang, F.; Lin, J.; Lemen, D.; Chakiath, C.; Mao, S.; Fazenbaker, C.; Zhong, H.; Harper, J.; Xu, W.; Patel, N.; Adams, L.; Vijayakrishnan, B.; Howard, P. W.; Marelli, M.; Wu, H.; Gao, C.; Read de Alaniz, J.; Christie, R. J., A Reactive Antibody Platform for One-Step Production of Antibody-Drug Conjugates through a Diels-Alder Reaction with Maleimide. Bioconjugate Chemistry 2019, 30 (9), 2340-2348.

37. Mees, M.; Haladjova, E.; Momekova, D.; Momekov, G.; Shestakova, P. S.; Tsvetanov, C. B.; Hoogenboom, R.; Rangelov, S., Partially Hydrolyzed Poly(n-propyl-2oxazoline): Synthesis, Aqueous Solution Properties, and Preparation of Gene Delivery Systems. Biomacromolecules 2016, 17 (11), 3580-3590.

38. Mees, M. A.; Hoogenboom, R., Full and partial hydrolysis of poly(2-oxazoline)s and the subsequent post-polymerization modification of the resulting polyethylenimine (co)polymers. Polymer Chemistry 2018, 9 (40), 4968-4978.

39. Luxenhofer, R., Polymers and nanomedicine: considerations on variability and reproducibility when combining complex systems. Nanomedicine (Lond) 2015, 10 (20), 310919.

40. Luxenhofer, R.; Sahay, G.; Schulz, A.; Alakhova, D.; Bronich, T. K.; Jordan, R.; Kabanov, A. V., Structure-property relationship in cytotoxicity and cell uptake of poly(2oxazoline) amphiphiles. J Control Release 2011, 153 (1), 73-82.

41. Gaertner, F. C.; Luxenhofer, R.; Blechert, B.; Jordan, R.; Essler, M., Synthesis, biodistribution and excretion of radiolabeled poly(2-alkyl-2-oxazoline)s. J Control Release 2007, 119 (3), 291-300.

42. Hahn, L.; Maier, M.; Stahlhut, P.; Beudert, M.; Flegler, V.; Forster, S.; Altmann, A.; Töppke, F.; Fischer, K.; Seiffert, S.; Böttcher, B.; Lühmann, T.; Luxenhofer, R., Inverse Thermogelation of Aqueous Triblock Copolymer Solutions into Macroporous Shear-Thinning 3D Printable Inks. ACS Applied Materials \& Interfaces 2020, 12 (11), 12445-12456.

43. Lübtow, M. M.; Mrlik, M.; Hahn, L.; Altmann, A.; Beudert, M.; Lühmann, T.; Luxenhofer, R., Temperature-Dependent Rheological and Viscoelastic Investigation of a Poly(2-methyl-2-oxazoline)-b-poly(2-iso-butyl-2-oxazoline)-b-poly(2-methyl-2-oxazoline)-

Based Thermogelling Hydrogel. Journal of functional biomaterials 2019, 10 (3).

44. Bauer, M.; Schroeder, S.; Tauhardt, L.; Kempe, K.; Schubert, U. S.; Fischer, D., In vitro hemocompatibility and cytotoxicity study of poly(2-methyl-2-oxazoline) for biomedical applications. Journal of Polymer Science Part A: Polymer Chemistry 2013, 51 (8), 1816-1821. 45. Yu, Y.; Zhang, Y.; Martin, J. A.; Ozbolat, I. T., Evaluation of cell viability and functionality in vessel-like bioprintable cell-laden tubular channels. J Biomech Eng 2013, 135 (9), 91011.

46. Hauptstein, J.; Böck, T.; Bartolf-Kopp, M.; Forster, L.; Stahlhut, P.; Nadernezhad, A.; Blahetek, G.; Zernecke-Madsen, A.; Detsch, R.; Jüngst, T.; Groll, J.; Teßmar, J.; Blunk, T., Hyaluronic Acid-Based Bioink Composition Enabling 3D Bioprinting and Improving Quality of Deposited Cartilaginous Extracellular Matrix. Adv Healthc Mater 2020, 9 (15), e2000737.

47. Farrugia, B. L.; Kempe, K.; Schubert, U. S.; Hoogenboom, R.; Dargaville, T. R., Poly(2-oxazoline) hydrogels for controlled fibroblast attachment. Biomacromolecules 2013, 14 (8), 2724-32.

48. Bott, K.; Upton, Z.; Schrobback, K.; Ehrbar, M.; Hubbell, J. A.; Lutolf, M. P.; Rizzi, S. C., The effect of matrix characteristics on fibroblast proliferation in 3D gels. Biomaterials 2010, 31 (32), 8454-64.

49. Murphy, C. M.; O'Brien, F. J., Understanding the effect of mean pore size on cell activity in collagen-glycosaminoglycan scaffolds. Cell Adh Migr 2010, 4 (3), 377-381. 
50. Geiger, F.; Rüdiger, D.; Zahler, S.; Engelke, H., Fiber stiffness, pore size and adhesion control migratory phenotype of MDA-MB-231 cells in collagen gels. PLoS One 2019, 14 (11), $\mathrm{e} 0225215$.

51. Arkenberg, M. R.; Moore, D. M.; Lin, C. C., Dynamic control of hydrogel crosslinking via sortase-mediated reversible transpeptidation. Acta Biomater 2019, 83, 83-95.

52. Seliktar, D.; Zisch, A. H.; Lutolf, M. P.; Wrana, J. L.; Hubbell, J. A., MMP-2 sensitive, VEGF-bearing bioactive hydrogels for promotion of vascular healing. J Biomed Mater Res A 2004, 68 (4), 704-16.

53. Wurst, H. P., DE), Larbi, Karima (Singapore, SG), Herrmann, Markus (Reutlingen, DE) CROSS-LINKING AGENTS FOR HYDROGELS THAT CONTAIN CLEAVABLE PEPTIDES AND SHORT-CHAIN POLYMERS. 2013.

54. Ritzer, J.; Lühmann, T.; Rode, C.; Pein-Hackelbusch, M.; Immohr, I.; Schedler, U.; Thiele, T.; Stübinger, S.; Rechenberg, B. v.; Waser-Althaus, J.; Schlottig, F.; Merli, M.; Dawe, H.; Karpíšek, M.; Wyrwa, R.; Schnabelrauch, M.; Meinel, L., Diagnosing peri-implant disease using the tongue as a 24/7 detector. Nature Communications 2017, 8 (1), 264.

55. Braun, A. C.; Gutmann, M.; Mueller, T. D.; Lühmann, T.; Meinel, L., Bioresponsive release of insulin-like growth factor-I from its PEGylated conjugate. Journal of Controlled Release 2018, 279, 17-28.

56. Braun, A. C.; Gutmann, M.; Ebert, R.; Jakob, F.; Gieseler, H.; Lühmann, T.; Meinel, L., Matrix Metalloproteinase Responsive Delivery of Myostatin Inhibitors. Pharm Res 2017, 34 (1), 58-72.

57. Dodt, K.; Lamer, S.; Drießen, M.; Bölch, S.; Schlosser, A.; Lühmann, T.; Meinel, L., Mass-Encoded Reporters Reporting Proteolytic Activity from within the Extracellular Matrix. ACS Biomaterials Science \& Engineering 2020, 6 (9), 5240-5253. 